Eur. J. Math. Anal. 2 (2022) 6

doi: $10.28924 /$ ada/ma.2.6

\title{
Solving Equilibrium Problem and Fixed Point Problem by Normal S-iteration Process in Hilbert Space
}

\author{
Shamshad Husain, Mohd Asad* \\ Department of Applied Mathematics, Faculty of Engineering and Technology, \\ Aligarh Muslim University, Aligarh, India \\ s_husain68@yahoo.com,masad19932015@gmail.com \\ ${ }^{*}$ Correspondence: masad19932015@gmail.com
}

\begin{abstract}
The main purpose of this paper is to find a common element in the solution set of equilibrium problem and fixed point problem of non-expansive mappings in the real Hilbert space with the help of normal S-iteration process. Also, under some acceptable assumptions, we prove the sequences induced by above stated process converge weakly to a point in the solution set of above stated problems. At the end, we give a numerical example to justify our work. The results studied in this work philosophize and boost some contemporary and known results in this direction.
\end{abstract}

\section{Introduction and Auxiliary results}

Everywhere in this paper except stated otherwise, let $H$ be a real Hilbert space equipped with inner product $\langle\cdot, \cdot\rangle$ and induced norm $\|\cdot\|$. Let $C$ be a non-empty closed and convex subset of $H$. We denote strong and weak convergence of a sequence $\left\{x_{n}\right\} \in H$ by the symbols $\rightarrow$ and $\rightarrow$ respectively.

Let $T: C \rightarrow H$ be a nonexpansive mapping. The so called fixed point problem for mapping $T$ is to find an element $p \in C$ such that

$$
T p=p
$$

Denote the set of solution of the problem (1) by $\operatorname{Fix}(T)=\{p \in C: T p=p\}$. $T$ is said to be nonexpansive iff

$$
\|T p-T q\|^{2} \leq\|p-q\|^{2}, \quad \forall p, q \in C .
$$

Received: 10 Dec 2021.

Key words and phrases. equilibrium problem; normal S-iteration; fixed point problem; Hilbert space; non-expansive mapping. 
In 2011, D.R. Sahu [4] studied problem (1) and proposed an iterative method known as Normal S-iteration Process which is defied as follows: Let $x_{1} \in C$ be chosen arbitrarily,

$$
\begin{aligned}
y_{n} & =\left(1-\alpha_{n}\right) x_{n}+\alpha_{n} T x_{n}, \\
x_{n+1} & =T y_{n}, \quad \forall n \geq 1,
\end{aligned}
$$

where $\left\{\alpha_{n}\right\} \subset(0,1)$. Under some acceptable conditions of $\left\{\alpha_{n}\right\}$, Sahu proved that the sequence $\left\{x_{n}\right\}$ induced by the algorithm (2) converges weakly to an element of solution set of problem (1). The performance of normal S-iteration process is much better than Mann and Picard iteration process for nonexpansive mappings(see [4], [5]).

Elsewhere, let $F: C \times C \rightarrow \mathbb{R}$ be a bifunction such that for all $p \in C, F(p, p)=0$. Then the so called equilibrium problem is to find $p \in C$ such that

$$
F(p, q) \geq 0, \quad \forall q \in C
$$

Denote the solution set of problem (3) by $E P(F)$. Problem (3) contains Nash equilibrium problems, fixed point problems, variational inequality problems, minimization problems and optimization problems as its special cases(see $[7,16])$.

In this paper, we consider a problem which is formulated as follows: Find $p \in C$, such that

$$
p \in \Omega:=\operatorname{Fix}(T) \cap E P(F) .
$$

In past few years, many researchers have found a common solution of problem (4) by various techniques(see [4], [3], [2], [12], [1], [14], [10]). Impelled and inspired by these approaches, the main objective of this paper is to find a common element in the solution set of problem (4) with the help of Normal S-iteration Process in the framework of real Hilbert space. Also we prove some weak convergence theorem under some acceptable conditions.

Now we define some basic auxiliary results which are very helpful throughout this work.

The metric projection $P_{C}$ from $H$ into $C$ is defined as: for any $p \in C$,

$$
\left\|p-P_{C}(p)\right\| \leq\|p-q\|, \quad \forall q \in C .
$$

It is to be noted that the metric projection is nonexpansive. Further for any $p \in H$ and $s \in C$,

$$
s=P_{C}(p) \Longleftrightarrow\langle p-s, s-q\rangle \geq 0, \quad \forall q \in C .
$$

A mapping $T$ is said to be monotone iff for all $p, q \in H$

$$
\langle T p-T q, p-q\rangle \geq 0 \text {. }
$$

Lemma 1.1. [9] Let $H$ be a Hilbert space. Then for all $p, q \in H$ and $\alpha \in[0,1]$ the followings hold:

(i) $\|p-q\|^{2}=\|p\|^{2}-\|q\|^{2}-2\langle p-q, q\rangle$;

(ii) $\|p+q\|^{2} \leq\|p\|^{2}+2\langle q, p+q\rangle$;

(iii) $\|\alpha p+(1-\alpha) q\|^{2}=\alpha\|p\|^{2}+(1-\alpha)\|q\|^{2}-\alpha(1-\alpha)\|p-q\|^{2}$. 
Assumption 1.1. [6] Let $F: C \times C \rightarrow \mathbb{R}$ be a bi-function satisfying the subsequent conditions:

(i) $F(p, p) \geq 0, \forall p \in C$;

(ii) $F$ is monotone, i.e. $F(p, q)+F(q, p) \leq 0, \forall p, q \in C$;

(iii) $F$ is upper semi continuous, i.e. for each $p, q, s \in C$,

$$
\lim _{t \rightarrow 0} \sup F(\lambda s+(1-\lambda) p, q) \leq F(p, q)
$$

(iv) For each fixed $p \in C$, the function $q \mapsto F(p, q)$ is convex and lower semi continuous;

Lemma 1.2. [7] Assume that the bi-function $F: C \times C \rightarrow \mathbb{R}$ satisfy the conditions of Assumption 1.1. Then for fixed $r>0$ and $p \in H$, there exists $s \in C$ such that

$$
F(q, p)+\frac{1}{r}\langle q-p, p-s\rangle \geq 0, \forall q \in C .
$$

Lemma 1.3. [12] Assume that the bi-function $F: C \times C \rightarrow \mathbb{R}$ satisfy the conditions of Assumption 1.1. If for $r>0$ and $p \in H$, defined a mapping $T_{r}^{F}: H \rightarrow C$ as follows:

$$
T_{r}^{F}(p)=\left\{s \in C: F(s, q)+\frac{1}{r}\langle q-s, s-p\rangle \geq 0, \forall q \in C\right\} .
$$

Then the followings hold:

(i) $T_{r}^{F}$ is non-empty and single valued.

(ii) $T_{r}^{F}$ is firmly non-expansive, i.e.,

$$
\left\|T_{r}^{F}(p)-T_{r}^{F}(q)\right\|^{2} \leq\left\langle T_{r}^{F}(p)-T_{r}^{F}(q), p-q\right\rangle \forall p, q \in H .
$$

(iii) $\operatorname{Fix}\left(T_{r}^{F}\right)=\operatorname{EP}(F)$.

(iv) $\mathrm{EP}(F)$ is closed and convex.

Lemma 1.4. [11] Let $\left\{a_{n}\right\}$ be a sequence of non negetive real numbers such that

$$
a_{n+1} \leq\left(1-\alpha_{n}\right) a_{n}+\alpha_{n} \delta_{n}+\gamma_{n}, \quad \forall n \geq 0,
$$

where $\alpha_{n} \in(0,1)$ and $\delta_{n} \subset \mathbb{R}$ satisfies the following conditions:

(i) $\sum_{n=0}^{\infty} \alpha_{n}=\infty$;

(ii) $\lim _{n \rightarrow \infty} \sup \delta_{n} \leq 0$.

(iii) $\gamma_{n} \geq 0(n \geq 1), \sum \gamma_{n}<\infty$.

Then $\lim _{n \rightarrow \infty} a_{n}=0$.

Lemma 1.5. [13] Let $C$ be a closed and convex subset of $H$ and $T: C \rightarrow C$ be a non-expansive mapping. Then

(i) $\operatorname{Fix}(T)$ is a closed and convex subset of $C$;

(ii) $I-T$ is demiclosed at 0 . 
Lemma 1.6. [8] Let $F: C \times C \rightarrow \mathbb{R}$ be a non linear bi-function satisfying the Assumption 1.1 and let $T_{r}^{F}$ be defined as above in Lemma 1.3. If for $r>0$, let $p, q \in H$ and $r_{1}, r_{2}>0$, Then

$$
\left\|T_{r_{2}}^{F}(q)-T_{r_{1}}^{F}(p)\right\| \leq\|q-p\|+\left|\frac{r_{2}-r_{1}}{r_{2}}\right|\left\|T_{r_{2}}^{F}(q)-q\right\| .
$$

Lemma 1.7. [15] Let $x_{n}$ and $y_{n}$ be two bounded sequences in a Banach space $X$ and let $\beta_{n}$ be a sequence in $[0,1]$ which satisfy the following conditions:

$$
0<\lim _{n \rightarrow \infty} \inf \beta_{n} \leq \lim _{n \rightarrow \infty} \sup \beta_{n}<1 .
$$

Suppose $x_{n+1}=\left(1-\beta_{n}\right) z_{n}+\beta_{n} x_{n}$ for all integers $n \geq 0$, and

$$
\lim _{n \rightarrow \infty} \sup \left(\left\|z_{n+1}-z_{n}\right\|-\left\|x_{n+1}-x_{n}\right\|\right) \leq 0, \quad \text { Then } \quad \lim _{n \rightarrow \infty}\left\|x_{n}-z_{n}\right\|=0 .
$$

\section{Main Result}

In this section we study and analyze Normal S-iteration process for solving equilibrium problem and fixed point problem for nonexpansive mapping and its convergence analysis.

Theorem 2.1. Let $C \subset H$ be a nonempty closed and convex subsets of $H$. Let $F: C \times C \rightarrow \mathbb{R}$ be a nonlinear bifunction satisfying Assumption 1.1. Let $T: C \rightarrow H$ be a nonexpansive mapping such that $\operatorname{Fix}(T) \neq$. Assume that $\Omega:=\operatorname{Fix}(T) \cap E P(F) \neq$. Let $\left\{x_{n}\right\}$ be a sequence defined as follows: Choose $x_{1} \in H$ arbitrarily,

$$
\begin{aligned}
y_{n} & =T_{r_{n}}^{F}\left(x_{n}\right), \\
z_{n} & =\left(1-\alpha_{n}\right) y_{n}+\alpha_{n} T y_{n}, \\
x_{n+1} & =T z_{n}, \quad \forall n \geq 1,
\end{aligned}
$$

where $\left\{\alpha_{n}\right\} \subset[0,1]$ and $\left\{r_{n}\right\} \subset(0, \infty)$ satisfying the following conditions:

C1: $\lim _{n \rightarrow \infty} \alpha_{n}=0, \quad \sum_{n=1}^{\infty} \alpha_{n}\left(1-\alpha_{n}\right)=\infty, \quad \sum_{n=1}^{\infty}\left|\alpha_{n}-\alpha_{n-1}\right|<\infty ;$

C2: $\lim _{n \rightarrow \infty} \inf r_{n}>0, \quad \sum_{n=0}^{\infty}\left|r_{n+1}-r_{n}\right|<\infty$;

Then the sequence $\left\{x_{n}\right\}$ induced by process (8) converges weakly to an element in $\Omega$.

Proof. Take $p \in \Omega$. Then by process (8), we obtain

$$
\begin{aligned}
\left\|x_{n+1}-p\right\| & =\left\|T z_{n}-p\right\| \leq\left\|z_{n}-p\right\|, \\
& \leq\left\|\left(1-\alpha_{n}\right) y_{n}+\alpha_{n} T y_{n}-p\right\|, \\
& \leq\left(1-\alpha_{n}\right)\left\|y_{n}-p\right\|+\alpha_{n}\left\|T y_{n}-p\right\|, \\
& \leq\left(1-\alpha_{n}\right)\left\|y_{n}-p\right\|+\alpha_{n}\left\|y_{n}-p\right\|, \\
& \leq\left\|y_{n}-p\right\| \leq\left\|T_{r_{n}}^{F}\left(x_{n}\right)-p\right\|, \\
& \leq\left\|x_{n}-p\right\| .
\end{aligned}
$$


By using mathematical induction, we have

$$
\left\|x_{n+1}-p\right\| \leq\left\|x_{n}-p\right\| \leq\left\|x_{1}-p\right\|, \quad \forall n \geq 1 .
$$

Hence the sequence $\left\{x_{n}\right\}$ is bounded and so are the sequences $\left\{y_{n}\right\},\left\{z_{n}\right\},\left\{T y_{n}\right\}$ and $\left\{T z_{n}\right\}$ are also bounded.

Let $M=\sup _{n} \geq 0\left\{\left\|y_{n}-x_{n}\right\|+\left\|x_{n}-q\right\|^{2}+\left\|T y_{n}\right\|+\left\|T z_{n}\right\|\right\}$.

Since $y_{n}=T_{r_{n}}^{F}\left(x_{n}\right)$ and $y_{n-1}=T_{r_{n-1}}^{F}\left(x_{n-1}\right)$, Then we obtain

$$
\begin{gathered}
F\left(y_{n}, q\right)+\frac{1}{r_{n}}\left\langle q-y_{n}, y_{n}-x_{n}\right\rangle \geq 0, \quad \forall q \in C, \\
F\left(y_{n-1}, q\right)+\frac{1}{r_{n-1}}\left\langle q-y_{n-1}, y_{n-1}-x_{n-1}\right\rangle \geq 0, \quad \forall q \in C .
\end{gathered}
$$

Replace $q$ by $y_{n}$ in (10) and $q$ by $q_{n-1}$ in (9) and adding them with the Assumption 1.1(ii),we obtain

$$
\left\langle y_{n}-y_{n-1}, \frac{y_{n-1}-x_{n-1}}{r_{n-1}}-\frac{y_{n}-x_{n}}{r_{n}}\right\rangle \geq 0,
$$

and hence

$$
\left\langle y_{n}-y_{n-1}, y_{n-1}-y_{n}-x_{n-1}-\frac{r_{n-1}}{r_{n}}\left(y_{n}-x_{n}\right)\right\rangle \geq 0 \text {. }
$$

This implies that by using Lemma 1.6

$$
\begin{aligned}
\left\|y_{n}-y_{n-1}\right\|^{2} & \leq\left\langle y_{n}-y_{n-1}, x_{n}-x_{n-1}+\left(1-\frac{r_{n-1}}{r_{n}}\right)\left(y_{n}-x_{n}\right)\right\rangle, \\
& \leq\left\|y_{n}-y_{n-1}\right\|\left\{\left\|x_{n}-x_{n-1}\right\|+\left|\frac{r_{n}-r_{n-1}}{r_{n}}\right|\left\|y_{n}-x_{n}\right\|\right\}, \\
\left\|y_{n}-y_{n-1}\right\| & \leq\left\|x_{n}-x_{n-1}\right\|+\left|\frac{r_{n}-r_{n-1}}{r_{n}}\right|\left\|y_{n}-x_{n}\right\|,
\end{aligned}
$$

From process (8)(C2), we have $\lim _{n \rightarrow \infty} \inf r_{n}>0$. Therefore there exists $r>0$ such that $r_{n}>r$ for large enough $n \in \mathbb{N}$. Then for $n \geq 1$,

$$
\left\|y_{n}-y_{n-1}\right\| \leq\left\|x_{n}-x_{n-1}\right\|+\frac{1}{r}\left|r_{n}-r_{n-1}\right| M .
$$

\section{Consider}

$$
\begin{aligned}
\left\|x_{n+1}-x_{n}\right\| & =\left\|T z_{n}-T z_{n-1}\right\| \leq\left\|z_{n}-z_{n-1}\right\|, \\
& \leq\left\|\left(1-\alpha_{n}\right) y_{n}+\alpha_{n} T y_{n}-\left(1-\alpha_{n-1}\right) y_{n-1}-\alpha_{n-1} T y_{n-1}\right\|, \\
& \leq \|\left(1-\alpha_{n}\right) y_{n}-\left(1-\alpha_{n}\right) y_{n-1}+\left(1-\alpha_{n}\right) y_{n-1}-\left(1-\alpha_{n-1}\right) y_{n-1} \\
& +\alpha_{n} T y_{n}-\alpha_{n} T y_{n-1}+-\alpha_{n} T y_{n-1}-\alpha_{n-1} T y_{n-1} \|, \\
& \leq\left(1-\alpha_{n}\right)\left\|y_{n}-y_{n-1}\right\|+2\left|\alpha_{n}-\alpha_{n-1}\right| M+\alpha_{n}\left\|y_{n}-y_{n-1}\right\|, \\
& \leq\left\|y_{n}-y_{n-1}\right\|+2\left|\alpha_{n}-\alpha_{n-1}\right| M .
\end{aligned}
$$


Using (11) and (12), we obtain

$$
\left\|x_{n+1}-x_{n}\right\| \leq\left\|x_{n}-x_{n-1}\right\|+\frac{1}{r}\left|r_{n}-r_{n-1}\right| M+2\left|\alpha_{n}-\alpha_{n-1}\right| M .
$$

By applying Lemma 1.4, we obtain

$$
\lim _{n \rightarrow \infty}\left\|x_{n+1}-x_{n}\right\|=0
$$

By using process (8)(C1)(C2) along with Lemma 1.7 and (13), we obtain

$$
\lim _{n \rightarrow \infty}\left\|x_{n}-z_{n}\right\|=0
$$

Furthermore, for any $p \in \Omega$, we have from process (8)

$$
\begin{aligned}
\left\|y_{n}-p\right\|^{2} & =\left\|T_{r_{n}}^{F}\left(x_{n}\right)-p\right\|^{2}, \\
& \leq\left\langle T_{r_{n}}^{F}\left(x_{n}\right)-T_{r_{n}}^{F}(p), x_{n}-p\right\rangle, \\
& \leq\left\langle y_{n}-p, x_{n}-p\right\rangle, \\
& \leq \frac{1}{2}\left\{\left\|y_{n}-p\right\|^{2}+\left\|x_{n}-p\right\|^{2}-\left\|x_{n}-y_{n}\right\|^{2}\right\}, \\
& \leq\left\|x_{n}-p\right\|^{2}-\left\|x_{n}-y_{n}\right\|^{2} .
\end{aligned}
$$

From convaxity of function $x \mapsto\|x\|^{2}$ and (16), we obtain

$$
\begin{aligned}
\left\|x_{n+1}-p\right\|^{2} & =\left\|T z_{n}-p\right\|^{2}, \\
& \leq\left\|z_{n}-p\right\|^{2}, \\
& \leq\left\|\left(1-\alpha_{n}\right) y_{n}+\alpha_{n} T y_{n}-p\right\|^{2}, \\
& \leq\left(1-\alpha_{n}\right)\left\|y_{n}-p\right\|^{2}+\alpha_{n}\left\|T y_{n}-p\right\|^{2}, \\
& \leq\left\|y_{n}-p\right\|^{2}, \\
& \leq\left\|x_{n}-p\right\|^{2}-\left\|x_{n}-y_{n}\right\|^{2} .
\end{aligned}
$$

And so,

$$
\begin{aligned}
\left\|x_{n}-y_{n}\right\|^{2} & \leq\left\|x_{n}-p\right\|^{2}-\left\|x_{n+1}-p\right\|^{2} \\
& \leq\left(\left\|x_{n}-p\right\|-\left\|x_{n+1}-p\right\|\right)\left(\left\|x_{n}-p\right\|+\left\|x_{n+1}-p\right\|\right) \\
& \leq\left\|x_{n}-x_{n+1}\right\|\left(\left\|x_{n}-p\right\|+\left\|x_{n+1}-p\right\|\right)
\end{aligned}
$$

Since the sequence $\left\{x_{n}\right\}$ is bounded and $\lim _{n \rightarrow \infty}\left\|x_{n+1}-x_{n}\right\|=0$. We have

$$
\lim _{n \rightarrow \infty}\left\|x_{n}-y_{n}\right\|=0
$$


Further,

$$
\begin{aligned}
\left\|x_{n+1}-p\right\|^{2} & =\left\|T z_{n}-p\right\|^{2}, \\
& \leq\left\|z_{n}-p\right\|^{2}, \\
& \leq\left\|\left(1-\alpha_{n}\right) y_{n}+\alpha_{n} T y_{n}-p\right\|^{2}, \\
& \leq\left(1-\alpha_{n}\right)\left\|y_{n}-p\right\|^{2}+\alpha_{n}\left\|T y_{n}-p\right\|^{2}-\alpha_{n}\left(1-\alpha_{n}\right)\left\|y_{n}-T y_{n}\right\|^{2}, \\
& \leq\left\|y_{n}-p\right\|^{2}-\alpha_{n}\left(1-\alpha_{n}\right)\left\|y_{n}-T y_{n}\right\|^{2}, \\
& \leq\left\|x_{n}-p\right\|^{2}-\left\|x_{n}-y_{n}\right\|^{2}-\alpha_{n}\left(1-\alpha_{n}\right)\left\|y_{n}-T y_{n}\right\|^{2},
\end{aligned}
$$

and so,

$$
\begin{aligned}
\alpha_{n}\left(1-\alpha_{n}\right)\left\|y_{n}-T y_{n}\right\|^{2} & \leq\left\|x_{n}-p\right\|^{2}-\left\|x_{n+1}-p\right\|^{2}-\left\|x_{n}-y_{n}\right\|^{2}, \\
& \leq\left\|x_{n}-x_{n+1}\right\|\left(\left\|x_{n}-p\right\|+\left\|x_{n+1}-p\right\|\right)-\left\|x_{n}-y_{n}\right\|^{2},
\end{aligned}
$$

using process (8)(C1), (14) and (17), we obtain

$$
\lim _{n \rightarrow \infty}\left\|y_{n}-T y_{n}\right\|=0 .
$$

consider

$$
\begin{aligned}
\left\|z_{n}-T z_{n}\right\| & \leq\left\|z_{n}-y_{n}\right\|+\left\|y_{n}-T y_{n}\right\|+\left\|T y_{n}-T z_{n}\right\|, \\
& \leq\left\|z_{n}-y_{n}\right\|+\left\|y_{n}-T y_{n}\right\|+\left\|y_{n}-z_{n}\right\| .
\end{aligned}
$$

By using (15) and (18), we obtain

$$
\lim _{n \rightarrow \infty}\left\|z_{n}-T z_{n}\right\|=0
$$

Since $\left\{x_{n}\right\}$ is bounded. There exists a subsequence $\left\{x_{n_{i}}\right\} \subset\left\{x_{n}\right\}$ such that $x_{n} \rightarrow \hat{p}$. Since $\lim _{n \rightarrow \infty}\left\|x_{n}-y_{n}\right\|=0$ and $\left\{y_{n}\right\}$ is bounded, this implies that $y_{n_{i}} \rightarrow \hat{p} \in C$. Now by (18) we have

$$
\left\|T y_{n_{i}}-y_{n_{i}}\right\| \rightarrow 0
$$

From (22) and Lemma 1.5, we conclude that $\hat{p} \in \operatorname{Fix}(T)$.

Next we prove that $\hat{p} \in E P(F)$. Since $y_{n}=T_{r_{n}}^{F}\left(x_{n}\right)$, we have

$$
F\left(y_{n}, q\right)+\frac{1}{r_{n}}\left\langle q-y_{n}, y_{n}-x_{n}\right\rangle \geq 0, \quad \forall q \in C .
$$

By using Assumption 1.1(ii), we obtain

$$
\frac{1}{r_{n}}\left\langle q-y_{n}, y_{n}-x_{n}\right\rangle \geq F\left(q, y_{n}\right)
$$

and so,

$$
\left\langle q-y_{n_{i}}, \frac{y_{n_{i}}-x_{n_{i}}}{r_{n_{i}}}\right\rangle \geq F\left(q, y_{n_{i}}\right) .
$$


Since $\frac{\left\|y_{n_{i}}-x_{n_{i}}\right\|}{r_{n_{i}}} \leq \frac{\left\|y_{n_{i}}-x_{n_{i}}\right\|}{r} \rightarrow 0$ and $y_{n_{i}} \rightarrow \hat{p}$, therefore by Assumption 1.1(iv), we obtain

$$
\lim _{n_{i} \rightarrow \infty} \inf F\left(q, y_{n_{i}}\right) \leq \lim _{n_{i} \rightarrow \infty}\left\langle q-y_{n_{i}}, \frac{y_{n_{i}}-x_{n_{i}}}{r_{n_{i}}}\right\rangle=0
$$

That is,

$$
F(q, \hat{p}) \leq 0, \quad \forall q \in C
$$

Further for any $\lambda \in(0,1)$ and $q \in C$, let $q_{\lambda}=\lambda q+(1-\lambda) \hat{p}$, then $q_{\lambda} \in C$ and so we have $F\left(q_{\lambda}, \hat{p}\right) \leq 0$. It follows from the Assumption 1.1 and $(24)$, that

$$
\begin{aligned}
0 & =F\left(q_{\lambda}, q_{\lambda}\right), \\
& \leq \lambda F\left(q_{\lambda}, q\right)+(1-\lambda) F\left(q_{\lambda}, \hat{p}\right), \\
& \leq \lambda F\left(q_{\lambda}, q\right) .
\end{aligned}
$$

This implies that $F\left(q_{\lambda}, q\right) \geq 0, \forall \lambda \in(0,1)$. Letting $\lambda \rightarrow 0^{+}$by Assumption 1.1, we have $F(\hat{p}, q) \geq 0, \quad \forall q \in C$. This implies that $\hat{p} \in E P(F)$ and hence $\hat{p} \in \Omega$. This completes the proof.

\section{Numerical Example}

Here we give numerical examples for supporting our main results. All codes are done by Matlab 2021 a.

Example 3.1. Set $H=\mathbb{R}$. Let $C=[0+\infty)$. Suppose $T: C \rightarrow H$, is defined by $T(p)=\frac{p}{3}$. It can be easily seen that, here $\operatorname{Fix}(T)=\{0\}$. Also, we define $F(s, q)=3 q^{2}+2 s q-5 s^{2}$, It is easy to check that $F$ satisfy the conditions of Assumption 1.1. So, for $r_{n}=r>0, T_{r}^{F}(p)$ is non-empty and single-valued for each $p \in C$. Hence for $r>0$, there exists $s \in C$ such that

$$
F(s, q)+\frac{1}{r}\langle q-s, s-p\rangle \geq 0 \forall q \in C,
$$

which is equivalent to

$$
3 r q^{2}+(s-p+2 r s) q+\left(p s-5 r s^{2}-s^{2}\right) \geq 0, \forall q \in C
$$

After solving the above inequality, we get $s=\frac{p}{1+8 r}$ for each $r>0$ i.e. $T_{r}^{F}(p)=\frac{p}{1+8 r}$ for each $r>0$. It can be easily seen that here $E P(F)=\{0\}$. This implies that $\Omega:=\operatorname{Fix}(T) \cap E P(F)=\{0\}$. Now, let us choose $r=\frac{1}{8}$, and $\left\{\alpha_{n}\right\}=\frac{1}{(n+6)} \cdot\left\{\alpha_{n}\right\}$ satisfy the conditions of main result.

Table. For different initial value, we present a table of iterations here. 


\begin{tabular}{ccc}
\hline \hline No. of iterations & $x_{0}=1$ & $x_{0}=-1$ \\
\hline 1 & 1.000000 & -1.000000 \\
2 & 0.150794 & -0.141923 \\
3 & 0.023038 & -0.020407 \\
4 & 0.003555 & -0.002964 \\
5 & 0.000553 & -0.000434 \\
6 & 0.000087 & -0.000064 \\
7 & 0.000014 & -0.000009 \\
8 & 0.000002 & -0.000001 \\
9 & 0.000000 & 0.000000 \\
\hline
\end{tabular}

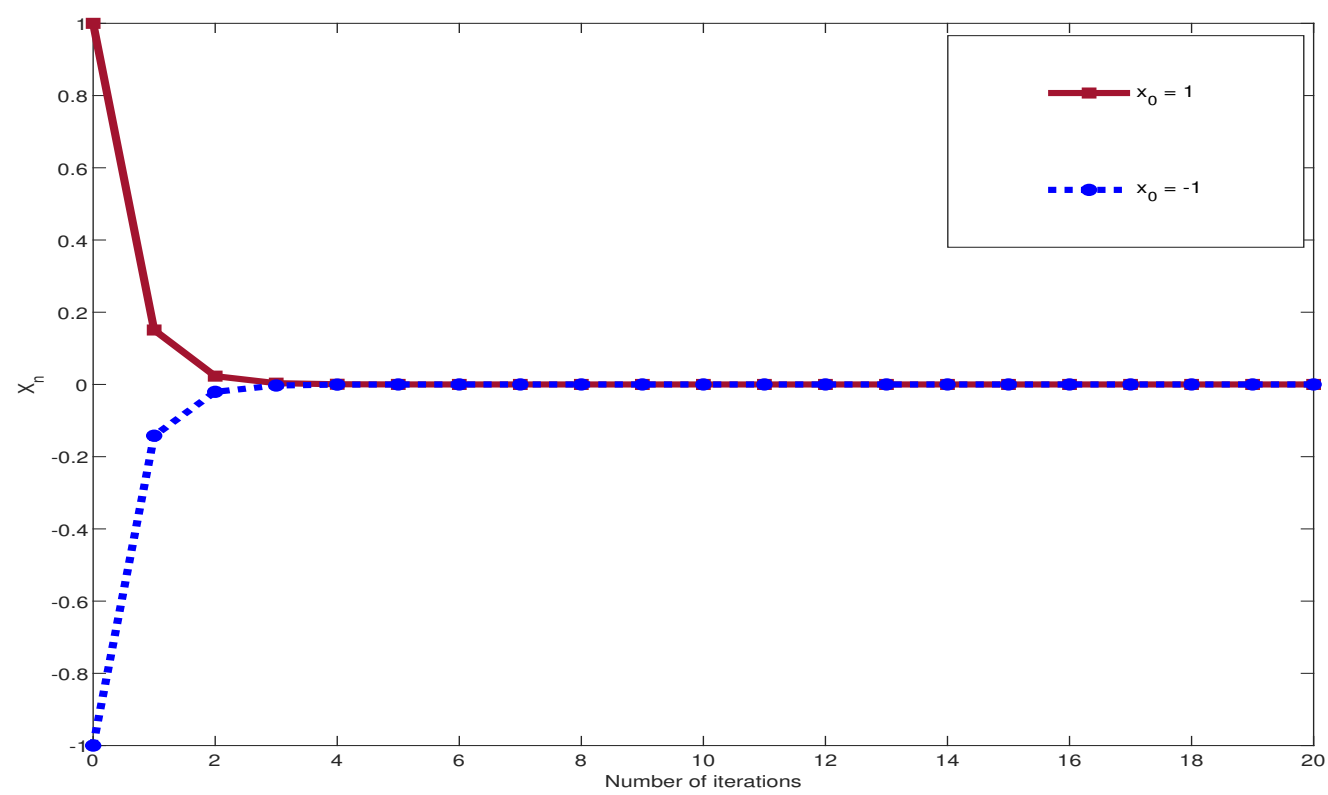

FIGURE 1. Graphical representation of sequence $\left\{x_{n}\right\}$ for different choices of initial value $x_{0}$.

\section{References}

[1] A. Moudafi, M. Théra, Proximal and dynamical approaches to equilibrium problems, in: M. Théra, R. Tichatschke (Eds.), Ill-Posed Variational Problems and Regularization Techniques, Springer Berlin Heidelberg, Berlin, Heidelberg, 1999: pp. 187-201. https://doi.org/10.1007/978-3-642-45780-7_12.

[2] A. Moudafi, Viscosity approximation methods for fixed-points problems, J. Math. Anal. Appl. 241 (2000) $46-55$. https://doi.org/10.1006/jmaa.1999.6615. 
[3] S.D. Flam, A.S. Antipin, Equilibrium programming using proximal-like algorithms, Math. Program. 78 (1996) $29-41$. https://doi.org/10.1007/BF02614504.

[4] D. R. Sahu, Applications of the S-iteration process to constrained minimization problems and split feasibility problems, Fixed Point Theory, 12 (2011) 187-204.

[5] D.R. Sahu, A. Pitea, M. Verma, A new iteration technique for nonlinear operators as concerns convex programming and feasibility problems, Numer. Algor. 83 (2020) 421-449. https://doi .org/10.1007/s11075-019-00688-9.

[6] H. Mahdioui, O. Chadli, On a system of generalized mixed equilibrium problems involving variational-like inequalities in Banach spaces: existence and algorithmic aspects, Adv. Oper. Res. 2012 (2012) 843486. https://doi.org/10. $1155 / 2012 / 843486$.

[7] E. Blum, W. Oettli, From optimization and variational inequalities to equilibrium problems. The Math. Students, 63 (1994), 123-145.

[8] F. Cianciaruso, G. Marino, L. Muglia, Y. Yao, A hybrid projection algorithm for finding solutions of mixed equilibrium problem and variational inequality problem, Fixed Point Theory Appl. 2010 (2010) 383740 https://doi.org/10.1155/2010/383740.

[9] H.H. Bauschke, P.L. Combettes, Convex analysis and monotone operator theory in hilbert spaces, 2nd ed. 2017, Springer International Publishing: Imprint: Springer, Cham, 2017. https://doi.org/10.1007/ $978-3-319-48311-5$.

[10] H.-K. Xu, Viscosity approximation methods for nonexpansive mappings, J. Math. Anal. Appl.298 (2004) $279-291$. https://doi.org/10.1016/j.jmaa.2004.04.059.

[11] H.-K. Xu, Iterative algorithms for nonlinear operators, J. Lond. Math. Soc. 66 (2002) 240-256. https://doi .org/ 10.1112/S0024610702003332.

[12] P.L. Combettes, S.A. Hirstoaga, Equilibrium programming in Hilbert spaces, J. Nonlinear Convex Anal. 6 (2005) 117-136.

[13] Q. Zhang, C. Cheng, Strong convergence theorem for a family of Lipschitz pseudocontractive mappings in a Hilbert space, Math. Computer Model. 48 (2008) 480-485. https://doi.org/10.1016/j.mcm.2007.09.014.

[14] S. Takahashi, W. Takahashi, Viscosity approximation methods for equilibrium problems and fixed point problems in Hilbert spaces, J. Math. Anal. Appl. 331 (2007) 506-515. https://doi.org/10.1016/j. jmaa. 2006.08.036.

[15] T. Suzuki, Strong convergence of Krasnoselskii and Mann's type sequences for one-parameter nonexpansive semigroups without Bochner integrals, J. Math. Anal. Appl. 305 (2005) 227-239. https://doi.org/10.1016/j. jmaa. 2004.11.017.

[16] S. Husain, N. Singh, $\Delta$-convergence for proximal point algorithm and fixed point problem in CAT(0) spaces, Fixed Point Theory Appl. 2019 (2019), 8. https://doi .org/10.1186/s13663-019-0658-3. 